\title{
OPEN Synthesis and in vitro PDT evaluation of red emission polymer dots (R-CPDs) and pyropheophorbide- $\alpha$ conjugates
}

\author{
Faiza Sajjad ${ }^{1}$, Yi-Jia Yan ${ }^{2 \bowtie}$, Davor Margetic ${ }^{3 凶}$ \& Zhi-Long Chen ${ }^{1 \bowtie}$
}

Carbon based polymer dots have piqued attention of researchers because of excellent biocompatibility, and good solubility. Most of the p-dots are able to generate ROS which is effective for photodynamic therapy for the treatment of cancer while some photosensitizers such as porphyrins possess some drawbacks such as hydrophobicity, and dark toxicity. Therefore in this study we conjugated red emission carbon based polymer with pyropheophorbide- $\alpha$ through amide condensation and $\pi-\pi$ stacking. One pot synthesis of the conjugate was successfully achieved. Their photophysiological properties were studied and structures were characterized by FT-IR, TEM and ${ }^{1} \mathrm{HNMR}$. pH- sensitivity of the conjugates was confirmed using fluorescence and UV-vis spectroscopy. Photo toxicity and dark toxicity of the prepared conjugates were evaluated in human esophageal cancer cell line (Eca-109). Hemocompatibility of the synthesized conjugates was evaluated and proved that the conjugates are safe to use for the treatment of tumor. Our results showed the PS doped p-dots had less dark toxicity and increased light toxicity as well as ROS generation was high as compared to precursor drug. Therefore, incorporation of $\mathrm{p}$-dots to porphyrin improved biocompatibility and enhanced the photodynamic effect.

Carbon-based fluorescence nanomaterials, such as carbon dots (CDs) and polymer dots (PDs), have stirred much attention, because of their enticing properties, e.g., high fluorescence ${ }^{1-4}$, excellent stability ${ }^{5,6}$ and good biocompatibility ${ }^{7-10}$. Although carbon dots and polymer dots both possess quasi-spherical shape but CDs possess inorganic carbon-based cores, while PDs have organic skeleton. CDs and PDs have been widely applied in the fields of sensing ${ }^{11-15}$, bioimaging ${ }^{16-19}$, and photodynamic/photothermal therapy (PDT/PTT) ${ }^{5,8,10,19,20}$ as alternatives for commercial semiconductor quantum dots and organic dyes. Different methods have been developed to produce CDs, including laser irradiation ${ }^{21,22}$, hydrothermal synthesis ${ }^{23,24}$, electrochemical etching ${ }^{25}$, and ultrasound and microwave-assisted syntheses ${ }^{26,27}$. These methods suffer from various limitations, such as high energy consumption and complex experiments. Moreover a high energy input would produce a large number of by-products ${ }^{26-29}$, which further require additional techniques for purification ${ }^{30}$. These problems limit the synthesis of CDs on a large scale. Additionally, their short-wavelength emission (400-550 nm) and poor water solubility further limit the use of semiconductor quantum dots and organic dyes in the fields of bioimaging and nanomedicine ${ }^{31-33}$.

Polymer dots (Pdots, PDs) have been developed as a brightly emissive nanoprobe used for bioimaging, diagnosis of the disease and drug delivery (therapy). Pdots are suitable for these different applications because of their excellent photophysical properties, high extinction coefficients, extraordinary particle brightness, and excellent photostability. Moreover, Pdots also possess good biocompatibility, tunable optical properties and surface properties and colloidal dimensions ${ }^{34}$. Most importantly, the carbon polymer dots (CPDs) have some drawbacks such as, short-wavelength emission, thus limit their application in bioimaging. Porphyrins belong to the family of tetrapyrrole has been extensively investigated as photosensitizers (PSs) for the treatment of tumors

${ }^{1}$ Department of Pharmaceutical Science \& Technology, College of Chemistry and Biology, DongHua University, Shanghai 201620, China. ${ }^{2}$ Shanghai Xianhui Pharmaceutical Co., Ltd, Shanghai 200433, China. ${ }^{3}$ Division of Organic Chemistry and Biochemistry, RuđerBošković Institute, Bijenička c. 54, 10000 Zagreb, Croatia. ${ }^{\bowtie}$ email: y_yan11@ sina.com; margetid@irb.hr; zlchen1967@qq.com 
using PDT. Though porphyrin-based molecules are the most commonly used PSs for PDT, many of them possess some drawbacks such as prolonged photosensitivity, poor solubility, dark toxicity, effective PDT and inadequate selectivity yield. To overcome these shortcomings, the incorporation of nanomaterials to porphyrins through self-assembly or covalent linkage is one of the effective methods ${ }^{35,36}$. Red emission carbon based polymer dots (R-CPDs) synthesized by $\mathrm{Xia}^{37}$ and his colleagues showed excellent resistance to photo bleaching, low cytotoxicity and $\mathrm{pH}$ sensitivity. Pyropheophorbide- $\alpha$ is a $(\mathrm{PPa})$ chlorophyll derivative with high ROS generation but owns certain drawbacks such as poor solubility, dark toxicity and low biocompatibility. One approach to overcome those problems is to functionalize the PS with compounds that have good compatibility with the tissue. So, to develop, compare and evaluate the efficient method to improve the overall efficacy of the photosensitizers used for PDT, in this study we synthesize R-CPDs with some modification and then conjugated R-CPDs to PPa through covalent and $\pi-\pi$ stacking. We also developed a facile one pot synthesis method for the incorporated conjugates which not only reduces the synthesis time and work but also decrease the toxicity of the PSs. Their photo-physiological properties and in vitro evaluation was performed to validate its potential use in biomedical application. The as-synthesized R-CPD conjugates not only accentuate the importance of functionalization of the nanomaterials but also provide a feasible approach to improve the therapeutic efficacy of the PSs.

\section{Material and methods}

PPa was acquired from Shanghai Xianhui Pharmaceutical Co., Ltd. All other chemicals and reagents were purchased from Sinopharm Chemical Reagent Co., Ltd and used without further purification. The blood was acquired from Zhenhu Medical Technology Co., Ltd. All solutions were freshly prepared with deionized water and all reactions were protected from sunlight. ${ }^{1} \mathrm{H}$ NMR spectra were recorded on a Bruker AMX-400 and chemical shifts $\delta(\mathrm{ppm})$ were referenced to TMS. UV-Vis absorption spectrum was recorded on an ultraviolet visible spectrophotometer (Model V-530, Japan). Fluorescence spectra were measured on a fluorescence spectrophotometer (FluoroMax-4, France). FTIR of the PD-hybrids was recorded on Perkin Elmer, spectrum-Two FT-IR and TEM images were taken from JEM-2100. Dynamic light scattering (DLS) experiments were done on a dynamic light scattering instrument BI-200SM.

Synthesis of R-CPDs. R-CPDs were synthesized according to the literature with some modifications ${ }^{37}$. Briefly, $10 \mathrm{mM}$ of p-phenylenediamine (pPD) and $100 \mathrm{mM} \mathrm{FeCl}_{3}$ was dissolved in $15 \mathrm{~mL}$ ultrapure water. The reaction mixture was heated at $120^{\circ} \mathrm{C}$ for $24 \mathrm{~h}$ in the air. The mixture was then filtered with a $0.22 \mu \mathrm{m}$ polyethersulfone membrane, dialyzed and the retentate/filtrate was lyophilized to obtain a powder.

Self-assembly of PDs to PPa (PPa-PD1). The non-covalent coordination $(\pi-\pi$ stacking) of PDs with pyropheophorbide- $\alpha$ was achieved following a method employed before. ${ }^{38}$. The PDs (30 mg in $1 \mathrm{~mL}$ water) and pyropheophorbide- $\alpha(36.0 \mathrm{mg}, 0.065 \mathrm{~mol})$ were dispersed in $2.0 \mathrm{~mL}$ dry DMF and sonicated for $4 \mathrm{~h}$. The mixture was then stirred at room temperature for $48 \mathrm{~h}$ followed by successive washing with ethanol, and deionized water by centrifugation. The product was finally washed with diethyl ether and dialyzed with DI water (Fig. 1i).

Covalent bonding between PPa and PDs (PPa-PD2). In the synthesis of R-CPD-PPa conjugate (PPaPD2), PPa (24.2 mg, $0.043 \mathrm{~mol})$ and HBTU (14.0 mg, 1.2 M) were dispersed in $2.0 \mathrm{~mL}$ dry DMF, $2.5 \mathrm{M}$ of DIPEA, and $30 \mathrm{mg}$ of R-CPDs in water was added and the mixture was stirred at room temperature for $12 \mathrm{~h}$. The product was then collected and successively washed with deionized water, ethanol by centrifugation and dialyzed with DI water to remove the residual byproduct (Fig. 1ii).

One-pot synthesis PPa.PD3. One-pot synthesis was successfully achieved by dissolving $10 \mathrm{mM}$ of $\mathrm{p}$-phenylenediamine, $100 \mathrm{mM} \mathrm{FeCl}_{3}$, and pyropheophorbide- $\alpha(2 \mathrm{mg}, 0.0036 \mathrm{~mol})$ in ultrapure water. The reaction was then stirred for $24 \mathrm{~h}$ at $120^{\circ} \mathrm{C}$. The mixture was then centrifuged several times to separate nano-conjugates from unreacted entities using ethanol. The product was filtered and dialyzed for two days (Fig. 1iii).

Absorption and emission spectra. Absorption spectra were recorded using ultraviolet-visible spectrophotometer. Spectra were collected from 300 to $800 \mathrm{~nm}$. Emission spectra were recorded using fluorescence spectrometer from 300 to $800 \mathrm{~nm}$ wavelength in $1 \mathrm{~nm}$ steps. Conjugates were dissolved in different solvents (DMSO, MeOH and DMF) at room temperature using quartz cuvettes.

Photo-bleaching assay. The photo stability of the synthesized conjugated was tested in DMSO. The absorption spectra of the nano-hybrids were measured at final concentrations of $10 \mu \mathrm{M}$ using $1 \mathrm{~cm}$ quartz cuvettes. Then, the solutions were irradiated with a semiconductor laser unit (PDT-670 nm), and the output power and discontinuous irradiation time were fixed to $5 \mathrm{~mW} / \mathrm{cm}^{2}$ and $120 \mathrm{~min}$. After irradiation, the absorbance of the conjugated drugs was measured again. The degree of photo-bleaching was calculated according to the equation: percent $(\%)=A_{t} / A_{0} \times 100$, where $A_{0}$ and $A_{t}$ represent the absorbance before and after irradiation, respectively, and $\mathrm{t}$ is the irradiation time $(10-120 \mathrm{~min})$. 
(i)

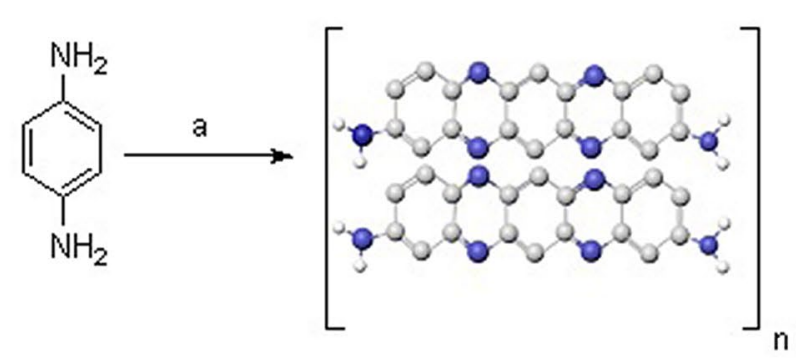

(ii)

(iii)<smiles>Nc1ccc(N)cc1</smiles>

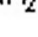

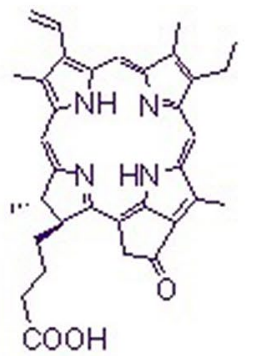

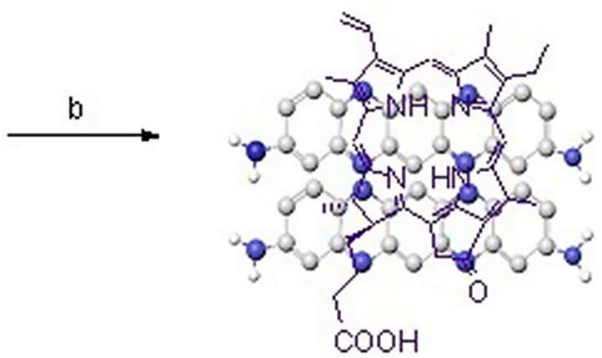

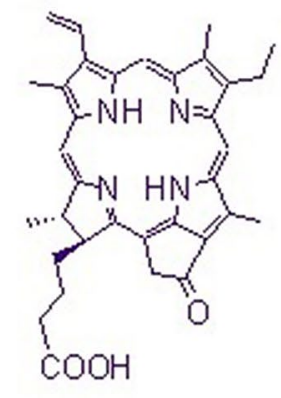
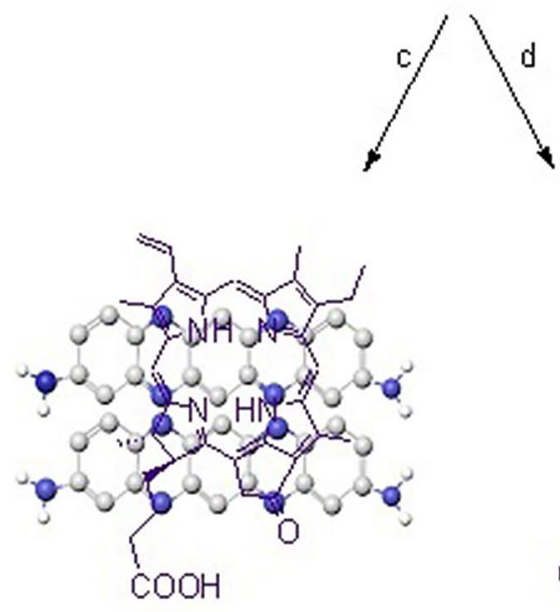

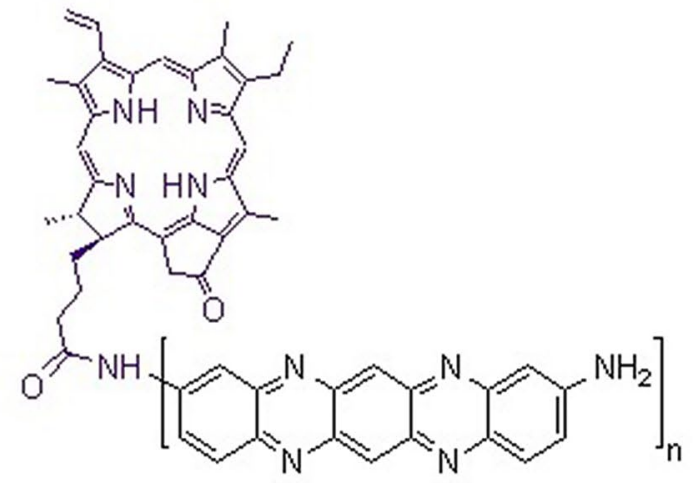

Figure 1. (i) Synthesis of R-CPDs and conjugates, (a) $\mathrm{FeCl} 2,80^{\circ} \mathrm{C}$, (ii) one pot synthesis of PPa-PD3, $120^{\circ} \mathrm{C}$, (iii) Conjugation of PPa-PD1 and PPa-PD2, (c) sonication of $48 \mathrm{~h}$, (d) DIPEA, HBTU.

Reactive oxygen species detection. The DPBF and each conjugate $(2 \mu \mathrm{M})$ was mixed at a 30 to 1 ratio in quartz cuvettes, which were irradiated every $5 \mathrm{~s}$ in the presence of oxygen using a $5 \mathrm{~mW} / \mathrm{cm}^{2} \mathrm{Nd}$ : YAG laser 
$(670 \mathrm{~nm})$ as the light source. The absorption spectra were measured by ultraviolet-visible spectrophotometer. Afterwards, the relative consumption of DPBF indicates the amount of singlet oxygen present. The rate of singlet oxygen generation calculated by the following equation described by

$$
\ln ([\mathrm{DPBF}] \mathrm{t} /[\mathrm{DPBF}] 0)=-\mathrm{kt}
$$

where $[\mathrm{DPBF}]_{\mathrm{t}}$ and $[\mathrm{DPBF}]_{0}$ are the concentrations of DPBF after and prior irradiation, respectively. Values of $\mathrm{k}$ are the rate of singlet oxygen generation and $\mathrm{t}$ is the time duration of irradiation ${ }^{39}$.

Cell culture. Human esophageal cancer cell line (Eca-109 cells) was purchased from Institute of Biochemistry and Cell Biology, CAS. The cells were maintained in RPMI medium, supplemented with 10\% FBS, 100 IU/ $\mathrm{mL}$ penicillin, and $100 \mathrm{mg} / \mathrm{mL}$ streptomycin at $37^{\circ} \mathrm{C}$ in a humidified atmosphere of $5 \% \mathrm{CO}_{2}$.

Cellular uptake of conjugates. Eca-109 cells were cultured and grown in 12 -well plates at $5 \times 10^{4}$ cells/ well. $24 \mathrm{~h}$ later, the medium was replaced with fresh medium containing $2 \mu \mathrm{M}$ compounds and incubated for an additional $24 \mathrm{~h}$. The cellular uptake of the compound was determined by a fluorescence spectrophotometer according to the method previously reported ${ }^{40}$.

Cellular uptake of the conjugates was observed using a fluorescence microscope. Cells were cultured over coverslips. After $24 \mathrm{~h}, 2 \mu \mathrm{M}$ of each conjugate was added and incubated overnight and then observed under a microscope ${ }^{41}$.

Cytotoxicity of conjugates. The conjugates in PBS were filtered and sterilized. A semiconductor laser was applied as a light source in PDT. Cells were cultured in 96 well plate at $5 \times 10^{4}$ cells $/ 100 \mu \mathrm{L}$ per well for $24 \mathrm{~h}$, treated with $0,1,2,3,4,5 \mu \mathrm{g} / \mathrm{mL}$ of PD-conjugates for dark toxicity and $0,0.1,0.25,0.5,0.75,1 \mu \mathrm{g} / \mathrm{mL}$ for phototoxicity. Light exposure was regulated by irradiation time, with the intensity of $2 \mathrm{~J} / \mathrm{cm}^{2}$, obtained with illumination times for $1 \mathrm{~min} 40 \mathrm{~s}$. Afterward $20 \mu \mathrm{L}$ MTT was added per well for $4 \mathrm{~h}$, and absorbance was measured with a microplate reader. The data was presented as mean \pm standard deviation $(\mathrm{SD})^{42}$. The cell viability was calculated according to the following formulation: cell viability $(\%)=$ OD experiment/OD control $\times 100 \%$. All experiments were carried out in triplicate. Cytotoxicity of the conjugates was also confirmed through florescence microscope using live and dead staining.

Hemolytic assay. To determine the effect of CD-conjugates on blood, the hemolytic assay was performed. Mouse blood was centrifuged $(16,000 \mathrm{rpm})$ for $5 \mathrm{~min}$ to collect erythrocyte. The obtained erythrocytes were washed with PBS three times and diluted. After that $500 \mu \mathrm{L}$ of diluted blood was added in tubes followed by the addition of $500 \mu \mathrm{L}$ of each conjugate, distilled water, PBS and incubated for $1 \mathrm{~h}$ at $37^{\circ} \mathrm{C}$. After incubation, each tube was centrifuged for $5 \mathrm{~min}$ at 16,000 rpm. The absorbance of collected supernatants was recorded at $545 \mathrm{~nm}$ with a microplate reader. PBS served as negative control while distilled water as a positive control. Hemolysis ratio was calculated using following formula ${ }^{43}$;

$$
\text { Hemolysis ratio }(\mathrm{HR})=[(\mathrm{Ap}-\mathrm{Ab}) /(\mathrm{At}-\mathrm{Ab})] \times 100 \%
$$

where Ap was the absorbance value of supernatant from each group, At was the absorbance value of the Triton $\mathrm{X}-100$ positive control and $\mathrm{Ab}$ was the absorbance value of DPBS. Each group contains three repeats.

Whole blood clotting. The effect of the conjugated drugs on blood clotting was evaluated. A volume of $50 \mu \mathrm{L}$ of recalcified whole-blood solution $\left(0.2 \mathrm{M} \mathrm{CaCl}_{2}, 10 \mathrm{mM}\right.$ in the blood) was added to drug $\left(37^{\circ} \mathrm{C}\right)$ in polypropylene tubes. Then, the tube was incubated at $37^{\circ} \mathrm{C}$ for $30 \mathrm{~s}, 60 \mathrm{~s}, 90 \mathrm{~s}$, and $120 \mathrm{~s}$, respectively. Blood was used as a control group. After pre-set time $1 \mathrm{~mL}$ of distilled water was added gently without disturbing the clot. The absorbance of the supernatant was recorded at $540 \mathrm{~nm}$ by using a microplate reader. Three replicates were performed.

$\mathrm{pH}$ sensitive response. The $\mathrm{pH}$ sensitivity of the synthesized nano-hybrids in different solutions (pH 4-8) were estimated. About $10 \mu \mathrm{M}$ concentration of each conjugate was dissolved in different $\mathrm{pH}$ solutions and emission spectra were taken through fluorescence spectrophotometer ${ }^{37}$. The $\mathrm{pH}$ sensitivity was also tested using UV-vis spectrophotometer following the method reported previously ${ }^{44}$.

Statistical analysis. Graphs were created by origin 8.0 (Graph Software, USA). All results are presented as mean \pm SD. Statistical significance was determined by unpaired two-tailed t tests or two-way analysis of variance. A $\mathrm{P}$ value of $<0.01$ and $<0.05$ was considered statistically significant. 
(a)

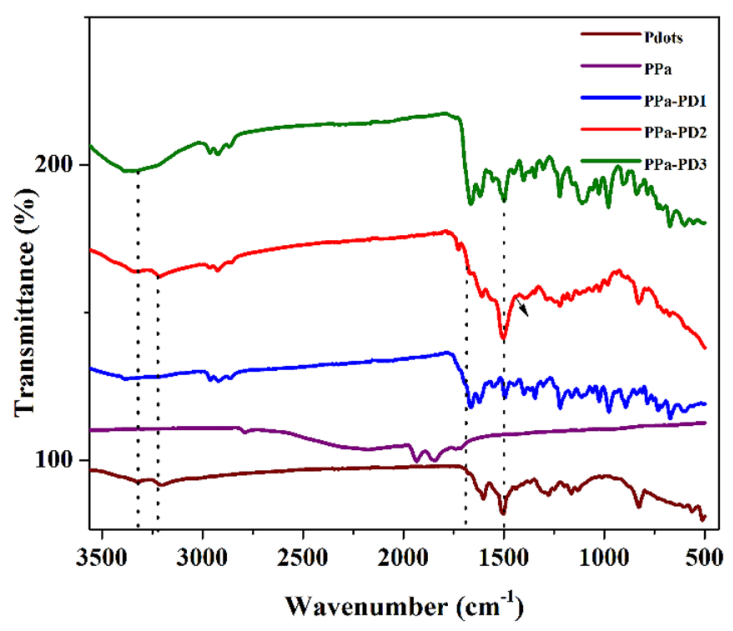

(b)

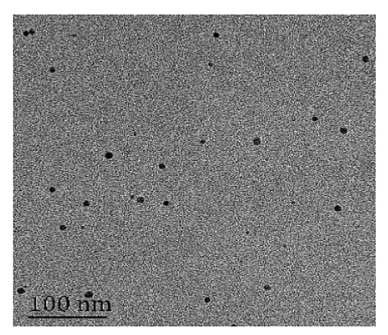

(c)

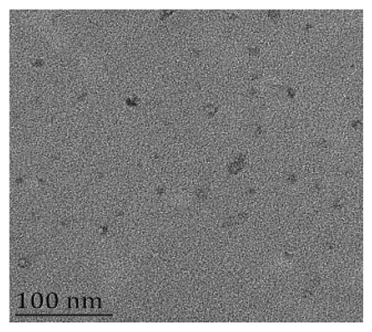

(d)

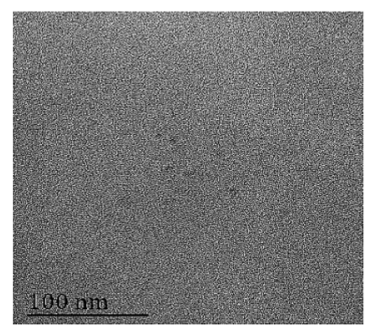

(e)

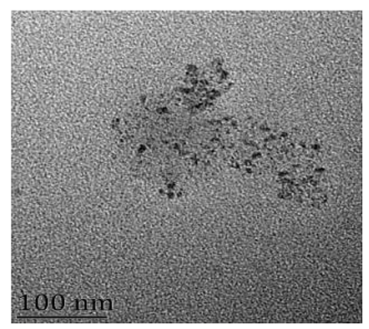

(f)

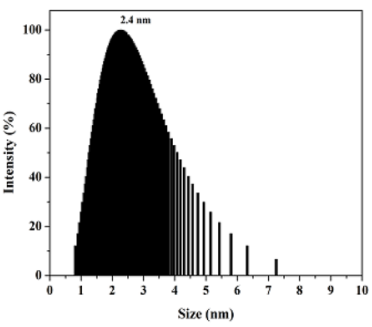

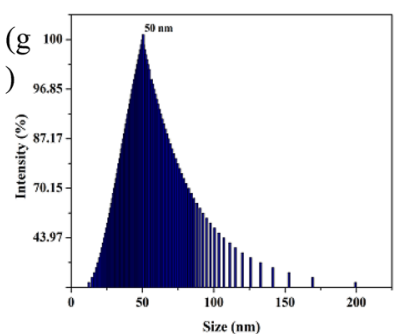
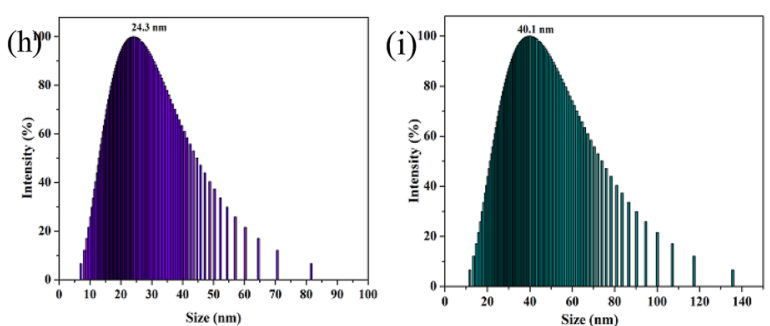

Figure 2. (a) FTIR of R-CPDs and conjugates. TEM images of (b) R-CPDs, (c) PPa-PD1, (d) PPa-PD2, (e) PPa-PD3. DLS of (e) R-CPDs, (f) PPa-PD1, (g) PPa-PD2, (i) PPa-PD3.

\section{Results and discussion}

Preparation of R-CPDs and PPa-PD conjugates. The R-CPDs were synthesized using $\mathrm{FeCl}_{3}$ as a catalyst to modulate polymerization of p-phenylenediamine in ultrapure water. The reaction mixture was stirred for $24 \mathrm{~h}$. The filtrate was dried to obtain dark color powder. The $\pi-\pi$ stacking of PDs with pyropheophorbide- $\alpha$ was achieved by dissolving PDs and pyropheophorbide- $\alpha$ in dry DMF. The mixture was sonicated for $4 \mathrm{~h}$ and then the reaction was continued for $48-72 \mathrm{~h}$. The nano-hybrid was then washed and centrifuged with ethanol, and deionized water.

For the covalent linkage between PPa and PDs, PPa (24.2 mg), HBTU and DIPEA were dispersed in $2.0 \mathrm{ml}$ dry DMF and, stirred for $2 \mathrm{~h}$. Then $30 \mathrm{mg}$ of R-CPDs solution was added and the mixture was stirred at room temperature for 12-24 h. The dark color product was collected after washing with ethanol. One pot approach was also used for the synthesis of the conjugated compound. PPa was dissolved in water, following the addition of $\mathrm{pPD}$ and $\mathrm{FeCl}_{3}$ and let it react for $24 \mathrm{~h}$. The unreacted species were then separated by centrifugation. The conjugated drug was filtered, and dialyzed to get pure product. 
(a)
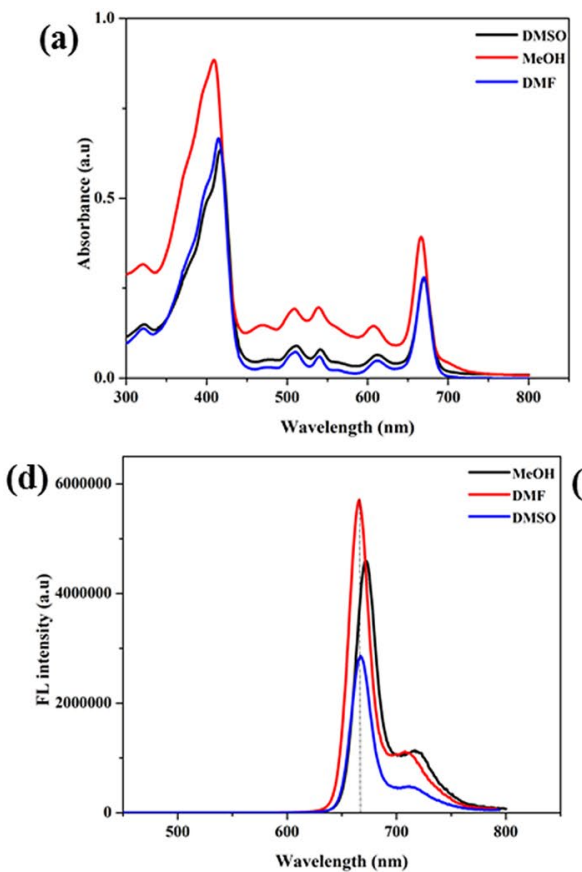

(b)

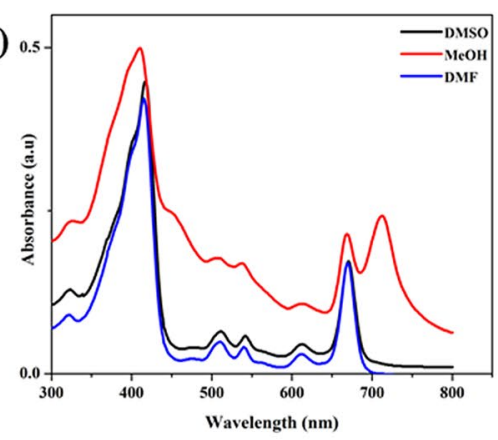

(e)

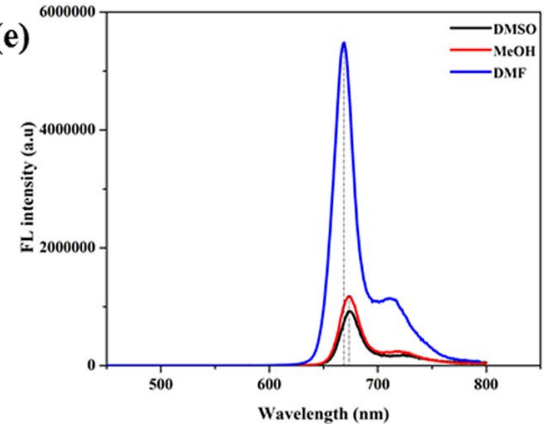

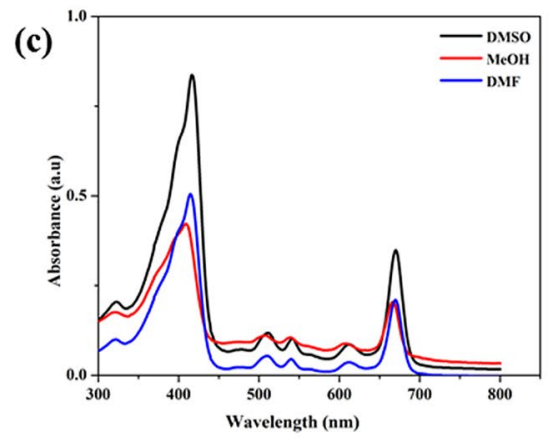

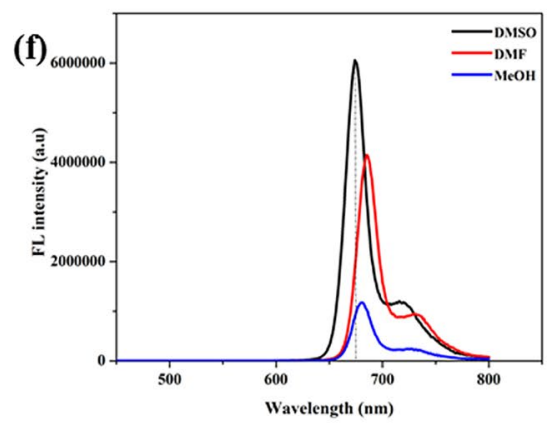

(g)

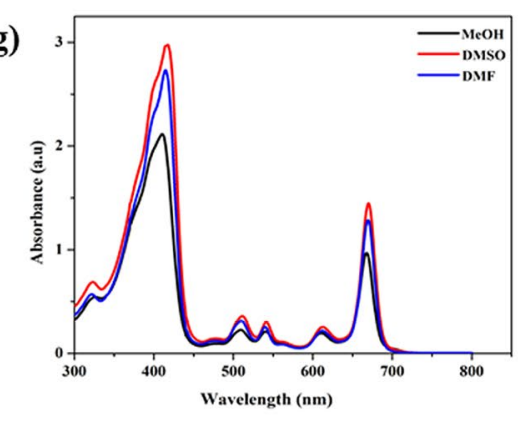

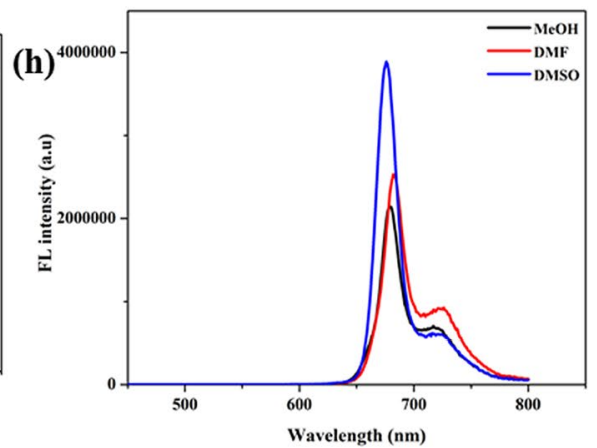

Figure 3. Absorbance of R-CPD conjugates (a) PPa-PD1, (b) PPa-PD2, (c) PPa-PD3, (d) Flourescence spectra of PPa-PD1, (e) PPa-PD2, (f) PPa-PD3, (g) PPa absorption spectra, (h) PPa fluorescence spectra.

Structural characterization. The synthesized R-CPDs and conjugates were characterized by FT-IR, TEM and ${ }^{1} \mathrm{HNMR}$. Figure 2a illustrates the FT-IR spectra of the hybrid compounds. The peak at $3200 \mathrm{~cm}^{-1}$ refers to the $\mathrm{N}-\mathrm{H}$ group while the peak around $1600 \mathrm{~cm}^{-1}$ predicts the presence of $\mathrm{C}=\mathrm{N} / \mathrm{C}=\mathrm{O}$. Retention of the bands in the region around 3000-3500 $\mathrm{cm}^{-1}$ suggests the presence of amide and hydroxyl groups in the prepared conjugates. The peak around $1200-1400 \mathrm{~cm}^{-1}$ refers to the presence of aliphatic compounds. The appearance of a peak in PPa-PD2 at $1433 \mathrm{~cm}^{-1}$ suggests the formation of an amide bond. The peak around $1600 \mathrm{~cm}^{-1}$ refers to the $\mathrm{C}=\mathrm{C} / \mathrm{C}=\mathrm{N}$ bond. The ${ }^{1} \mathrm{HNMR}$ of $\mathrm{PPa}$ and $\mathrm{PD}$ conjugates confirmed the structures of nano-hybrids. The incorporated drugs were further confirmed by ${ }^{1}$ HNMR spectroscopy (Fig S1a-e). The peak around $7.4 \mathrm{ppm}$ (PPa-PD2) refers to the formation of an amide bond. The spectra showed peaks at 7-9 ppm which indicates the aromatic $\left(\mathrm{sp}^{2}\right)$ hydrogen atoms of the precursor drug. To further strengthen the structural studies of the PDconjugates transmission electron microscopy was done. The morphology and size of the nano-hybrids is shown in Fig. 2b-e. The sizes of the conjugates were confirmed by Dynamic light scattering (DLS). An increase in the distribution frequency of the diameter of each integrated compound showed the successful incorporation of PDs to the porphyrin molecule. The huge increase in size after conjugation suggests the aggregation of porphyrins as, porphyrins tend to aggregate when interacted with nanoparticles due to the adsorption of porphyrins into the nanoparticles (Fig. $2 \mathrm{f}-\mathrm{i}$ ). In porphyrins, aggregation by $\pi-\pi$ stacking is common ${ }^{38}$. It was observed that the size of PPa-PD3 was smaller than PPa-PD1, which suggests that the temperature might affect the aggregation of the porphyrins when interacted with nanoparticles. 

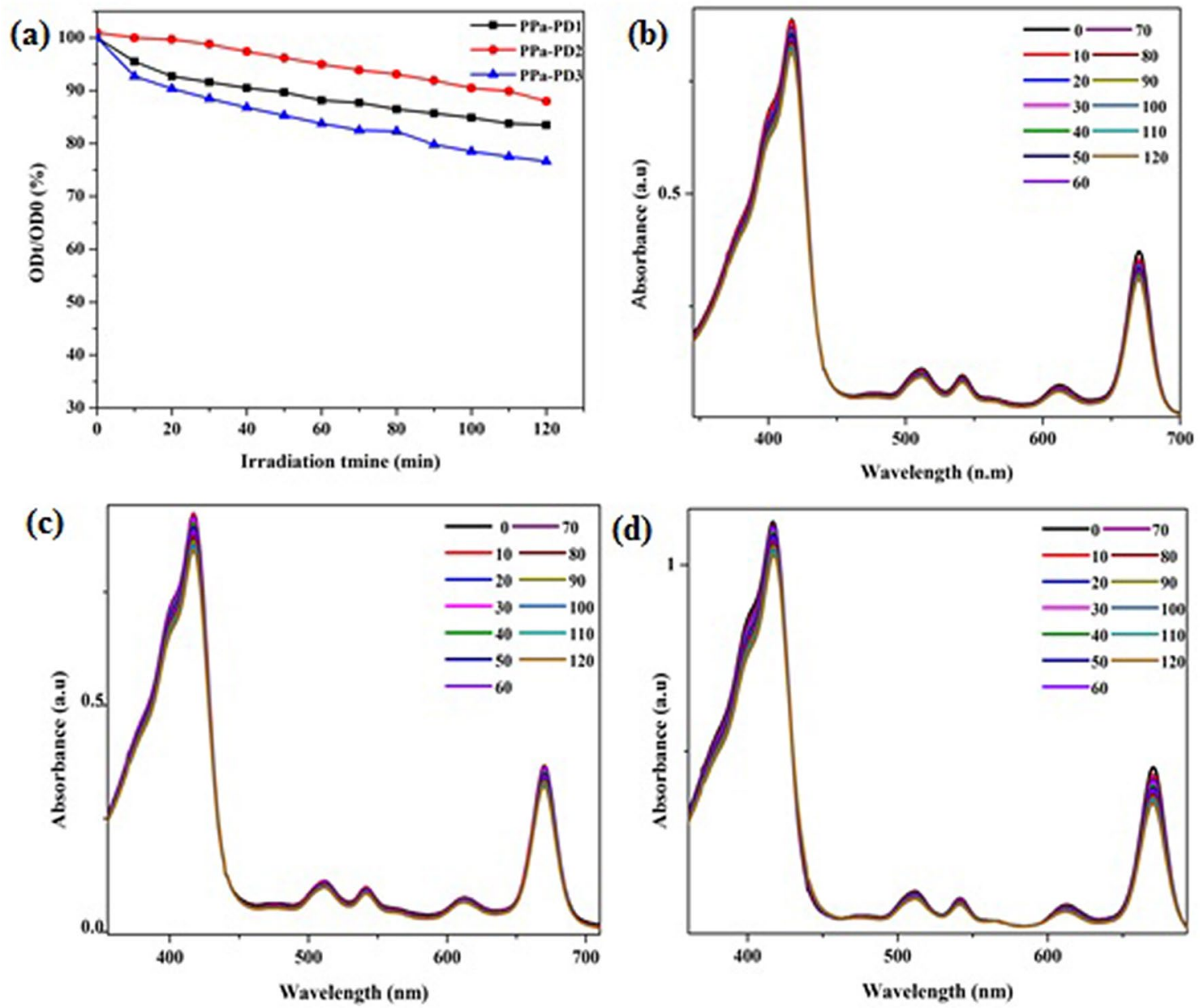

Figure 4. (a) Photostability of the conjugates in DMSO, (b) PPa-PD1, (c) PPa-PD2, (d) PPa-PD3.

Photophysical studies. Absorption and emission spectra of photo luminescent compounds are important for diagnosis and treatment. Figure $3 \mathrm{a}-\mathrm{c}$ illustrated the absorption spectra of the PD-nanocomposites in different solvents. The synthesized hybrids showed a broad absorption from 400 to $670 \mathrm{~nm}$. The absorbance of the conjugates had a shift of $2 \mathrm{~nm}$ from precursor drug (Fig. $3 \mathrm{~g}$ ) which is $668 \mathrm{~nm}$ in different solvents while for PPa-PD2 the absorbance peak at $713 \mathrm{~nm}$ was observed which suggest the successful conjugation. The shift in the peak might be because of the high polarity of methanol. Porphyrins as well as R-CPDs are known for their photoluminescence. The fluorescence emission spectra of conjugates synthesized following different mechanisms were depicted in Fig. 3d-f. Some organic drugs possess solvatochromic effect, different fluorescence in different solvents ${ }^{45,46}$. These results depicted the solvent-dependent fluorescence emission of PPa-PD hybrids. Fluorescence peak was observed at $675 \mathrm{~nm}$ in DMSO while in the PPa-PD3 peak was shifted to $685 \mathrm{~nm}$ which was at $681 \mathrm{~nm}$ in PPa (Fig. 3h) when dissolved in DMF.

Photostability. One of the concerning issues with the photosensitive drugs is stability. The rate of photostability plays an important role in photodynamic therapy ${ }^{47}$. The photostability of the drugs was evaluated and, the OD values of maximum absorption showed no significant change with the irradiation time after $2 \mathrm{~h}$ of irradiation indicated that the conjugated PDs were stable and, the structure of compounds did not change after $120 \mathrm{~min}$ of laser treatment (Fig. 4). One eminent advantage of the covalently PPa-incorporated Pdots is their excellent stability that solves the photosensitizer leaching problem.

ROS generation. Singlet oxygen generation by photosensitizers is another important factor affecting PDT. Different scavengers of singlet oxygen are present nowadays. We used 1,3-diphenylisobenzofuran (DPBF) to determine the rate of ROS generation ${ }^{48}$. Upon laser irradiation, the excited nano-hybrids generated singlet oxygen. The singlet oxygen produced as a result of irradiation then induced tumor destruction. Our studies showed that the incorporation of PDs to $\mathrm{PPa}$ increased the singlet oxygen as compared to the precursor compound. The 
(a)
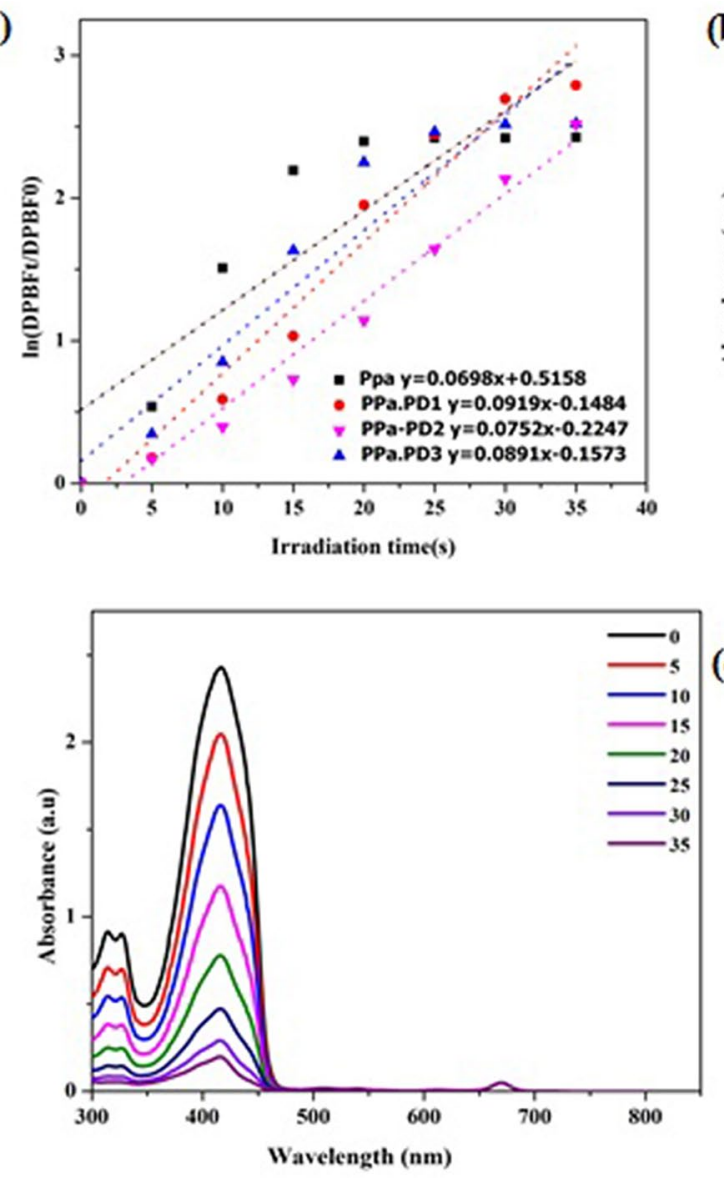

(b)

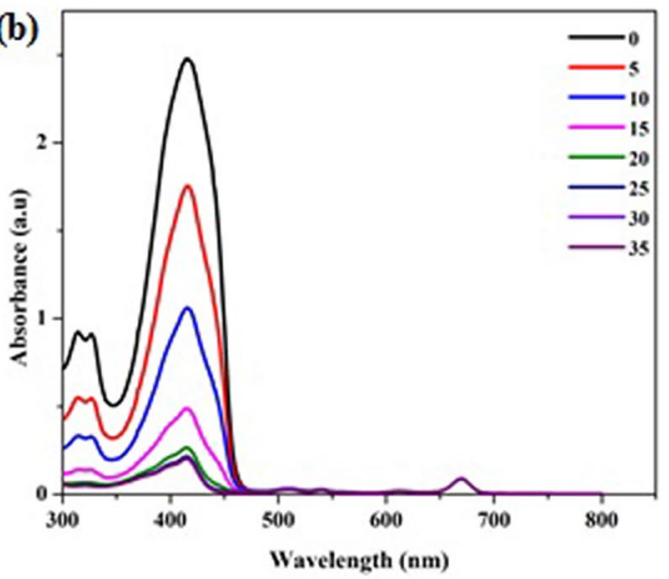

(d)

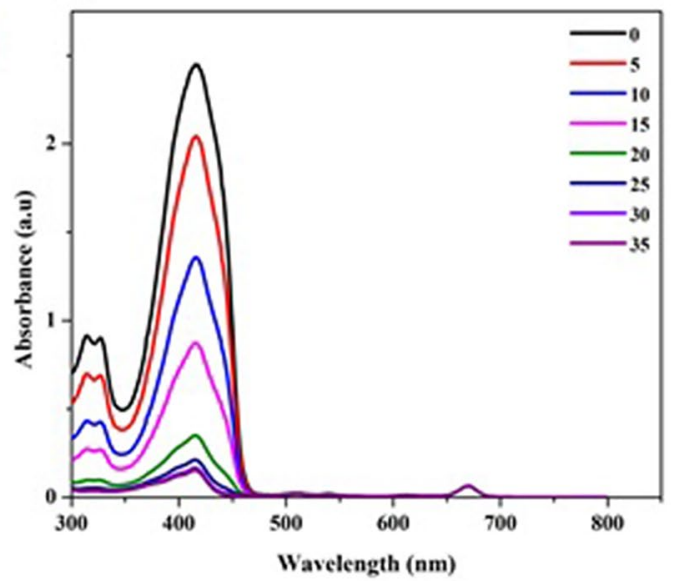

Figure 5. (a) ROS generation in nano-hybrids. Photodecomposition of DPBF in (b) PPa-PD1, (c) PPa-PD2, (d) PPa-PD3.

decrease in absorbance of DPBF was remarkable in each hybrid drug. Each PD hybrid showed fast bleaching of DPBF (Fig. 5).

Dark and light toxicity. The increased ${ }^{1} \mathrm{O}_{2}$ yield and good photostability of PPa-Pdots make them a promising candidate for photodynamic cancer treatment. The dark and light toxic effect of the conjugated drugs was studied using MTT assay and fluorescence microscopy. We used cellular staining to investigate the photodynamic toxicity induced by the PPa-PDs. The staining of the cells showed apparent cell death after treatment with Pdots and light irradiation as compared to the control groups in the absence of Pdots incubation or light irradiation (Fig. 6a). The results of the MTT assay further confirmed the PDT induced toxicity of the conjugates. As illustrated in Fig. 6b,c the cellular viabilities decreased apparently as the Pdot concentration increased. The cell viability showed a negligible decreased after incubation with Pdots at high concentration $(5 \mu \mathrm{g} / \mathrm{mL})$, indicating the good biocompatibility of the Pdots in the absence of light. The low dark toxicity of the Pdots is one of the important characteristics of their practical applications. The cell staining results together with the cell viability studies indicated that PPa-Pdots could function as a potent nanoparticle photosensitizer for cancer cell damage due to the efficient ROS generation.

Cellular uptake. To investigate the cellular uptake of pdot hybrids, the fluorescence imaging was performed on Eca-109. After incubation for $24 \mathrm{~h}$, Eca-109 cells showed fluorescence when observed under a fluorescence microscope indicating the accumulation of the drug in cells (Fig. $7 \mathrm{a}-\mathrm{c}$ ). The cellular uptake was further confirmed by fluorescence analysis as shown in Fig. $7 \mathrm{~d}$. After $24 \mathrm{~h}$ of incubation, maximum absorption was observed. So, the synthesized nano-hybrids can be absorbed by the cells and produced efficient PDT actions.

Hemocompatiblity. In vitro hemolysis assay is a universal method to evaluate the hemocompatibility of materials $\mathrm{s}^{49}$. The hemolysis ratios of the conjugates were tested. The macroscopical color of centrifugally obtained 
(a)

\section{Control}
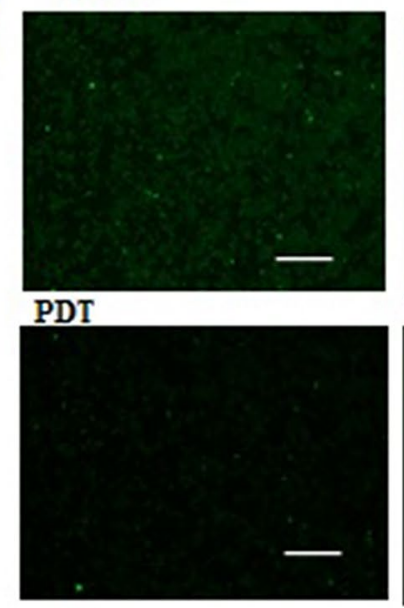

\section{PPa-PDl}
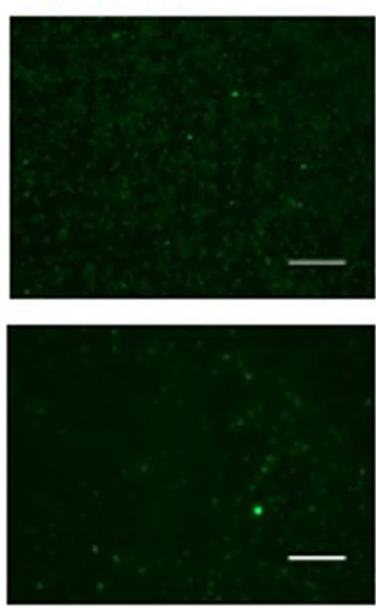

(b)

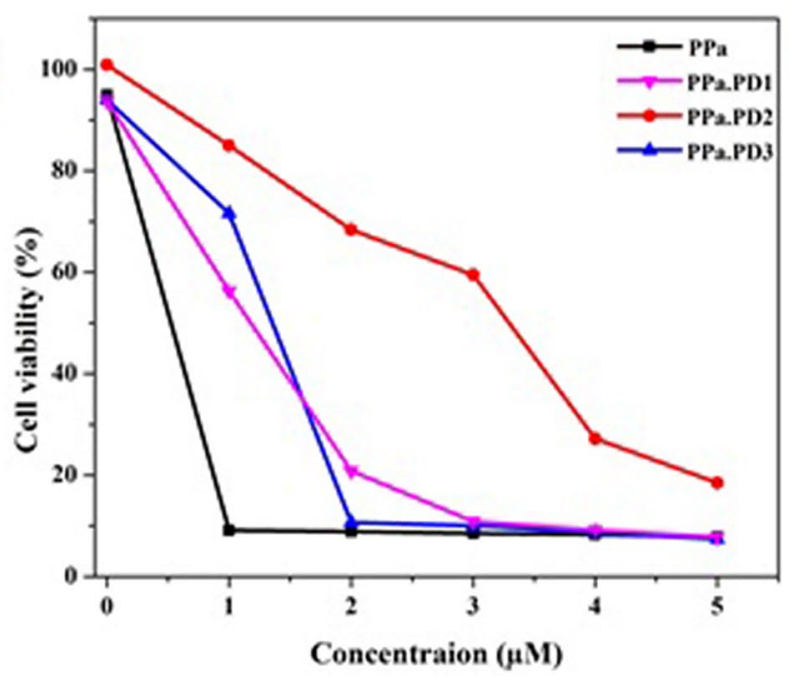

PPa-PD2
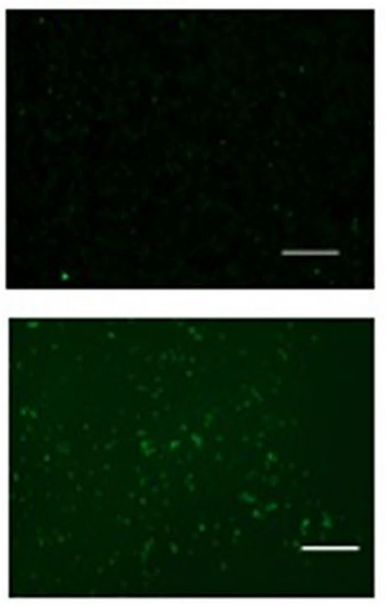

PPa-PD3
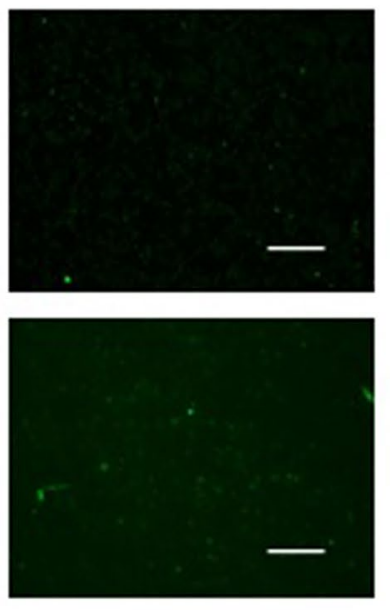

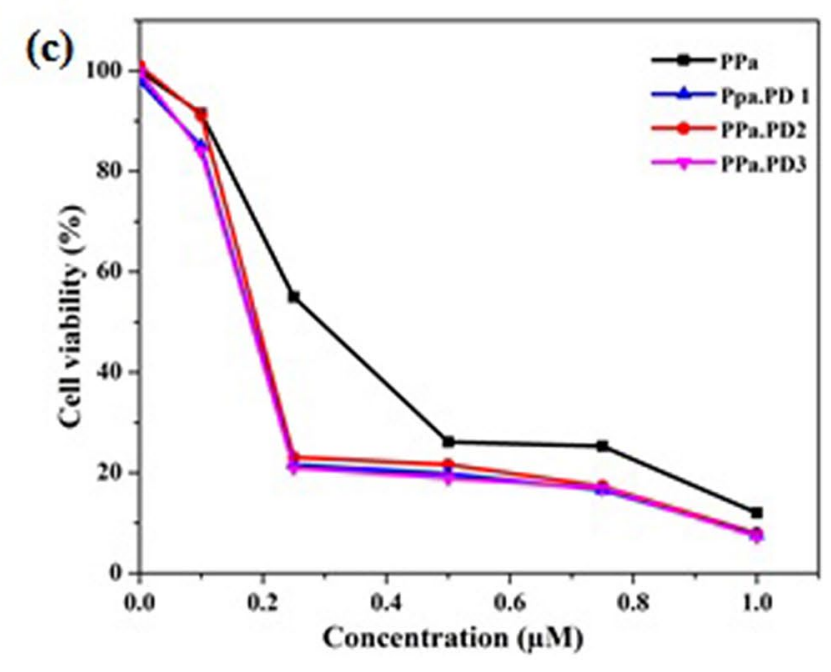

Figure 6. (a) Cell viability of Eca-109 after PDT. (b) MTT assay dark toxicity, (c) Phototoxicity. Scale bar $200 \mu \mathrm{m}$.

supernatants for drug groups, and control groups was shown in Fig S2a. The color of the supernatant obtained depicted the hemolytic ratio of the erythrocytes when treated with $5 \mu \mathrm{g}$ of conjugates and their precursors. Drug groups presented light yellow similar to the PBS control group, while the positive group was bright red. The quantitative data of the hemolysis ratio was shown in Table 1. PPa-PD conjugates showed a low hemolysis ratio as compared to the precursor molecule revealing its best hemocompatibility. If the hemolysis rate is below $5 \%$, medical materials will be considered as non-hemolysis according to national biological safety ${ }^{50}$. The results of the samples were all less than $5 \%$, conforming to the national biological material hemolysis rate security specified requirements. The blood clotting was tested using each conjugate. Our results showed that during first $30 \mathrm{~s}$ OD of the control was higher than the drug groups but at the end of $120 \mathrm{~s}$ the OD of the control and hybrid drug was the same while its higher in case of precursor molecule, suggesting that the conjugation of PDs to PS help to maintain hemostasis of the body fluids. The OD of the PPa-PD1 and PPa-PD3 were much lower after $120 \mathrm{~s}$ suggesting that the drugs were hemocompatible and did not affect the normal hemostatis of the body (Fig.S2b). The results of the hemolysis ratio and clotting test suggested that the incorporation of the p-dots with the organic drugs could improve hemocompatibility.

$\mathrm{pH}$ sensitivity of R-CPD conjugates. As Xia et al. reported, the synthesized R-CPDs possess $\mathrm{pH}$ sensitivity which is an important characteristic for its application is biomedicines. PPa-PD hybrids were supposed 
(a)
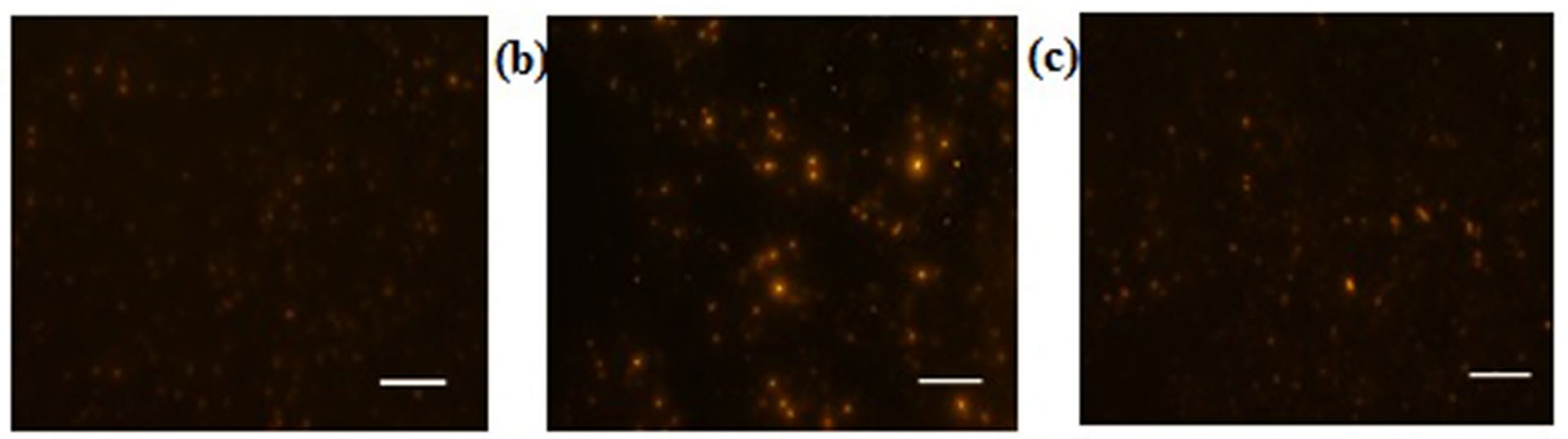

(d)

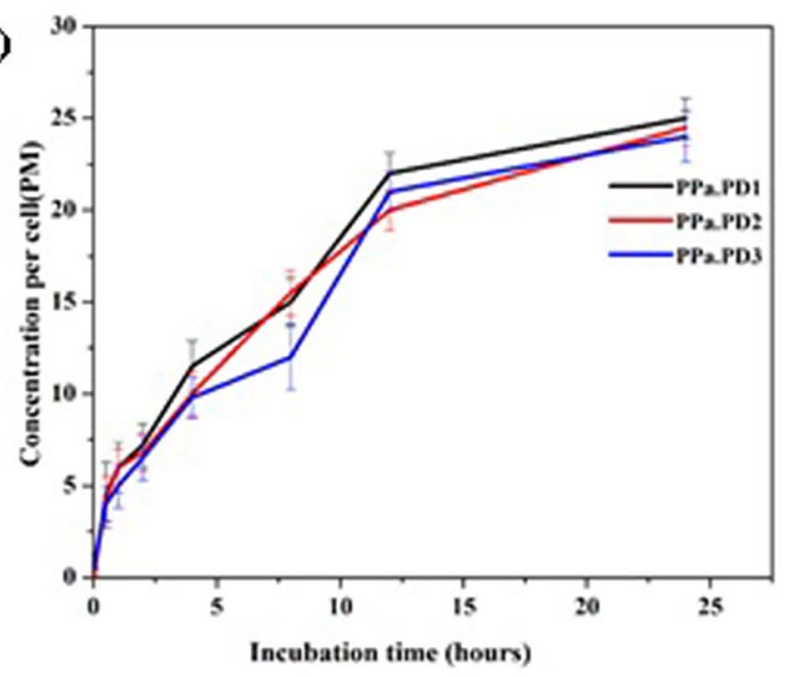

Figure 7. PD conjugates uptake by Eca-109 (a) PPa-PD1, (b) PPa-PD2, (c) PPa-PD3 (d) Cellular uptake. Scale bar $200 \mathrm{~nm}$.

\begin{tabular}{|l|l|l|}
\hline Samples & Optical density at $\mathbf{5 4 5} \mathbf{~ n m}$ & Hemolysis ratio (\%) \\
\hline PBS & $0.0710 \pm 0.0113$ & 0 \\
\hline Water & $1.5682 \pm 0.0197$ & 100 \\
\hline PPa & $0.0928 \pm 0.0169$ & 1.47 \\
\hline PPa-PD1 & $0.0859 \pm 0.01202$ & 0.99 \\
\hline PPa-PD2 & $0.0877 \pm 0.01463$ & 1.11 \\
\hline PPa-PD3 & $0.0912 \pm 0.00155$ & 1.34 \\
\hline
\end{tabular}

Table 1. Hemolysis caused by different conjugates $(5 \mu \mathrm{g})$. Standard deviation is calculated from three samples.

to exhibit $\mathrm{pH}$ sensitivity. To obtain proof for this assumption, the effects of a broad range of $\mathrm{pH}$ from 4 to 8 on the optical properties of the nanohybrids were investigated. As shown in Fig. 8, the PD-hybrids displayed strong fluorescence signals at $674 \mathrm{~nm}$ in a neutral environment, and the peak was shifted to $677 \mathrm{~nm}$ in a solution of 4 $\mathrm{pH}$ (Fig. 8b). The FL intensity showed a change with $\mathrm{pH}$ in a range of 5 to 6 in case of $\pi-\pi$ stacking which was consistent with the results of Xia et al. While FL intensity decreased at $8 \mathrm{pH}$ when p-dots were covalently bonded with $\mathrm{PPa}$ might be due to the deprotonation of the porphyrin in an alkaline environment. $\mathrm{pH}$ sensitive response was evaluated using UV-vis absorption spectra. Figure $8 \mathrm{~d}-\mathrm{f}$ showed the absorbance's of pdot-hybrids from $\mathrm{pH}$ 4 to 8 . The peak at 417 and $702(713) \mathrm{nm}$ decreased in the $\mathrm{pH}$ solution of 7 while it increased in acidic solution 

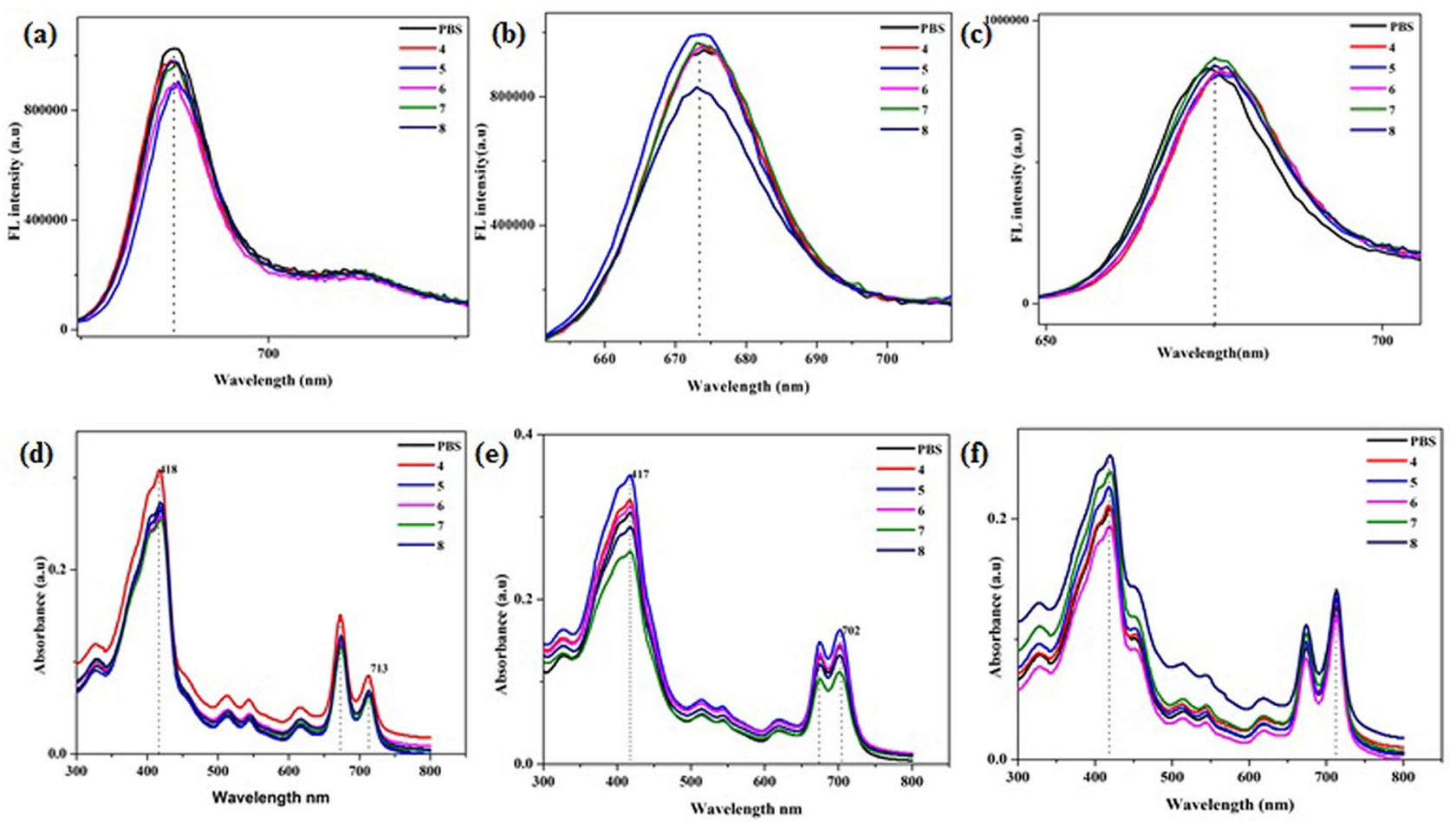

Figure 8. PL Spectra of conjugates at various $\mathrm{pH}$ values (a) PPa-PD1, (b) PPa-PD2, (c) PPa-PD3: UV-Vis spectra in different $\mathrm{pH}$ solutions (e) PPa-PD1, (f) PPa-PD2, (g) PPa-PD3.

(PPa-PD1, PPa-PD2). A slight shift of 2-3 nm was observed in each drug solution at $417 \mathrm{~nm}$ in basic solutions. Therefore, the $\mathrm{pH}$-responsive nanoprobe based on PDs might be suitable for $\mathrm{pH}$ sensing.

\section{Conclusion}

In short, we synthesized red emission carbon based polymers dots and later incorporated them with a widely used photosensitizer PPa through $\pi-\pi$ stacking and covalent bonding. We also developed one pot facile synthesis of the hybrid drug. The synthesized hybrids were characterized by TEM, FT-IR, ${ }^{1} \mathrm{HNMR}, \mathrm{UV}-\mathrm{Vis}$ and, fluorescence spectrophotometry. The absorbance of the PD conjugates was found to be increased which confers the successful conjugation. The resulting Pdots possessed excellent stability as well as yields high singlet oxygen. Facile one pot synthesis of the nanohybrids was achived at low temperature. The one pot synthesis was an efficient method to synthesize the conjugate as this method not only reduced the synthesis time but also enhanced the PDT efficacy of the hybrid. DLS results propose that the temperature has effect on the aggregation of the conjugates. The cytotoxicity and photodynamic effects of the Pdots were evaluated in Eca-109 cells by MTT assay. The dark toxicity of the photosensitizer dope p-dots was reduced as compared to pyropheophorbide- $\alpha$, indicating that the hybrid drugs could efficiently damage cancer cells due to the high-yield ${ }^{1} \mathrm{O}_{2}$ generation. Accordingly, PPa-PDs could be accumulated in Eca-109 cells, leading to the remarkable photodynamic therapeutic effect under laser irradiation. The hemolysis ratio of nanohybrids suggested that the synthesized composite drugs were biocompatible. The nano-composites displayed a change in fluorescence signal in different $\mathrm{pH}$ solutions suggests that the conjugate inherit the $\mathrm{pH}$ sensitivity of the PDs. The synthesized nanohybrids possessed good solubility, high ROS generation and low dark toxicity. This study will offer a new perspective in the design of hybrid photosensitizers and may advance biomedical research using such biomaterials and nanomedicine.

Received: 9 November 2020; Accepted: 19 April 2021

Published online: 11 May 2021

\section{References}

1. Rong, Y. et al. Multicolor fluorescent semiconducting polymer dots with narrow emissions and high brightness. ACS Nano 7 , 376-384 (2013). 
2. Wang, H. et al. Excitation wavelength independent visible color emission of carbon dots. Nanoscale 9, 1909-1915 (2017).

3. Yuan, F. L. et al. $53 \%$ Efficient red emissive carbon quantum dots for high color rendering and stable warm white-light emitting diodes. Adv. Mater. 29, 1702910 (2017).

4. Chen, X. Z. et al. Multicolor super-resolution fluorescence microscopy with blue and carmine small photoblinking polymer dots. ACS Nano 11, 8084-8091 (2017).

5. Yan, Y. B. et al. Chen Systematic bandgap engineering of graphene quantum dots and applications for photocatalytic water splitting and $\mathrm{CO} 2$ reduction. ACS Nano 12, 3523-3532 (2018).

6. Yang, T. et al. Ultrastable near-infrared conjugated-polymer nanoparticles for dually photoactive tumor inhibition. Adv. Mater. 29, 1700487 (2017).

7. Essner, J. B. et al. Pee-dots: Biocompatible fluorescent carbon dots derived from the upcycling of urine. Green Chem. 18, 243-250 (2016).

8. Zhang, J. F. et al. Biocompatible D-A semiconducting polymer nanoparticle with light-harvesting unit for highly effective photoacoustic imaging guided photothermal therapy. Adv. Funct. Mater. 27, 1605094 (2017).

9. Qu, S. N. et al. A biocompatible fluorescent ink based on water-soluble luminescent carbon nanodots. Angew. Chem. Int. Ed. 51, 12215-12218 (2012).

10. Geng, J. L. et al. Biocompatible conjugated polymer nanoparticles for efficient photothermal tumor therapy. Small 11, 1603-1610 (2015).

11. Gao, W. L. et al. Carbon dots with red emission for sensing of Pt2+, Au3+ and $\mathrm{Pd} 2+$ and their bio-applications in vitro and in vivo. ACS Appl. Mater. Interfaces 10, 1147-1154 (2018).

12. Kalytchuk, S. et al. Carbon dot nanothermometry: Intracellular photoluminescence lifetime thermal sensing. ACS Nano 11, 1432-1442 (2017).

13. Shi, H. F. et al. Ultrasmall phosphorescent polymer dots for ratiometric oxygen sensing and photodynamic cancer therapy. $A d v$. Funct. Mater. 24, 4823-4830 (2014).

14. Nie, H. et al. Carbon dots with continuously tunable full-color emission and their application in ratiometric pH sensing. Chem. Mater. 26, 3104-3112 (2014).

15. Shi, W., Li, X. H. \& Ma, H. M. A tunable ratiometric $\mathrm{pH}$ sensor based on carbon nanodots for the quantitative measurement of the intracellular $\mathrm{pH}$ of whole cells. Angew. Chem. Int. Ed. 51, 6432-6435 (2012).

16. Liu, X. L. et al. Nitrogen-doped carbon quantum dot stabilized magnetic iron oxide nanoprobe for fluorescence, magnetic resonance, and computed tomography triple-modal in vivo bioimaging. Adv. Funct. Mater. 26, 8694-8706 (2016).

17. Zhu, S. J. et al. Highly photoluminescent carbon dots for multicolor patterning, sensors, and bioimaging. Angew. Chem Int. Ed. 52, 3953-3957 (2013).

18. Ding, D. et al. Bright far-red/near-infrared conjugated polymer nanoparticles for in vivo bioimaging. Small 9, 3093-3102 (2013).

19. Yu, J. B., Rong, Y., Kuo, C. T., Zhou, X. H. \& Chiu, D. T. Recent advances in the development of highly luminescent semiconducting polymer dots and nanoparticles for biological imaging and medicine. Anal. Chem. 89, 42-56 (2017).

20. Ge, J. C. et al. Red-emissive carbon dots for fluorescent, photoacoustic, and thermal theranostics in living mice. Adv. Mater. 27, 4169-4177 (2015).

21. Hu, S. L. et al. One-step synthesis of fluorescent carbon nanoparticles by laser irradiation. J. Mater. Chem. 19, 484-488 (2009).

22. Li, X. Y. et al. Preparation of carbon quantum dots with tunable photoluminescence by rapid laser passivation in ordinary organic solvents. Chem. Commun. 47, 932-934 (2011).

23. Li, Y. et al. An electrochemical avenue to green-luminescent graphene quantum dots as potential electron-acceptors for photovoltaics. Adv. Mater. 23, 776-780 (2011).

24. Liu, H., He, Z., Jiang, L. P. \& Zhu, J. J. Microwave-assisted synthesis of wavelength-tunable photoluminescent carbon nanodots and their potential applications. ACS Appl. Mater. Interfaces 7, 4913-4920 (2015).

25. Zhuo, S. J., Shao, M. W. \& Lee, S. T. Upconversion and downconversion fluorescent graphene quantum dots: Ultrasonic preparation and photocatalysis. ACS Nano 6, 1059-1064 (2012).

26. Schneider, J. et al. Molecular fluorescence in citric acid-based carbon dots. J. Phys. Chem. C 121, 2014-2022 (2017).

27. Shi, L. et al. Carbon dots with high fluorescence quantum yield: The fluorescence originates from organic fluorophores. Nanoscale 8, 14374-14378 (2016).

28. Song, Y. et al. Investigation from chemical structure to photoluminescent mechanism: A type of carbon dots from the pyrolysis of citric acid and an amine. J. Mater. Chem. C 3, 5976-5984 (2015).

29. Kasprzyk, W., Bednarz, S. \& Bogdal, D. Luminescence phenomena of biodegradable photoluminescent poly (diol citrates). Chem. Commun. 49, 6445-6447 (2013).

30. Essner, J. B., Kist, J. A., Polo-Parada, L. \& Baker, G. A. Artifacts and errors associated with the ubiquitous presence of fluorescent impurities in carbon nanodots. Chem. Mater. 30, 1878-1887 (2018).

31. Jiang, K. et al. Red, green, and blue luminescence by carbon dots: Full-color emission tuning and multicolor cellular imaging. Angew. Chem. Int. Ed. 54, 5360-5363 (2015).

32. Hola, K. et al. Carbon dots-emerging light emitters for bioimaging, cancer therapy and optoelectronics. Nano Today 9, 590-603 (2014).

33. Song, Y. B., Zhu, S. J. \& Yang, B. Bioimaging based on fluorescent carbon dots. RSC Adv. 4, 27184-27200 (2014).

34. Jiangbo, Yu., Rong, Yu., Kuo, C.-T., Zhou, X.-H. \& Chiu, D. T. Recent advances in the development of highly luminescent semiconducting polymer dots and nanoparticles for biological imaging and medicine. Anal. Chem. 89(1), 42-56 (2017).

35. Lovell, J. F. et al. Porphysome nanovesicles generated by porphyrin bilayers for use as multimodal biophotonic contrast agents. Nat. Mater. 10, 324-332 (2011).

36. Zou, Q. et al. Biological photothermal nanodots based on self-assembly of peptide-porphyrin conjugates for antitumor therapy. J. Am. Chem. Soc. 139, 1921-1927 (2017).

37. Xia, J. et al. Synthesis of highly stable red-emissive carbon polymer dots by modulated polymerization: From the mechanism to application in intracellular pH imaging. Nanoscale 10, 22484 (2018).

38. Managa, M., Achadu, O. J. \& Nyokong, T. Photophysical studies of graphene quantum dots-Pyrene-derivatized porphyrins conjugates when encapsulated within Pluronic F127 micelles. Dyes Pigments 148, 405-416 (2018).

39. Tang, W., Xu, H., Kopelman, R. \& Philbert, M. A. Photodynamic characterization and in vitro application of methylene bluecontaining nanoparticle platforms. Photochem. Photobiol. 81, 242-249 (2015).

40. Zhang, L. J. et al. Antitumor activity evaluation of meso-tetra (pyrrolidine substituted) pentylporphinmediated photodynamic therapy in vitro and in vivo. J. Photochem. Photobiol. B 163, 224-231 (2016).

41. Zhang, Y. et al. Temperature-dependent cell death patterns induced by functionalized gold nanoparticle photothermal therapy in melanoma cells. Sci. Rep. 8, 8720 (2018).

42. Borik, R. M., Nagwa, M. F., Sherifa, M. \& Magdy, S. A. Design, synthesis, anticancer evaluation and docking studies of novel heterocyclic derivatives obtained via reactions involving curcumin. Molecules 23, 1398 (2018).

43. Zhao, X. et al. Injectable antibacterial conductive nanocomposite cryogels with rapid shape recovery for noncompressible hemorrhage and wound healing. Nat. Commun. 9, 2784 (2018).

44. Huo, Z. et al. A two-photon fluorescence, carbonized polymer dot (CPD)-based, wide range $\mathrm{pH}$ nanosensor; a view from the surface state. Nanoscale 10, 1039 (2020). 
45. Dong, J., Solntsev, K. M. \& Tolbert, L. M. Solvatochromism of the green fluorescence protein chromophore and its derivatives. J. Am. Chem. Soc. 128, 12038-12039 (2006).

46. Terenziani, F. et al. Charge instability in quadrupolar chromophores: Symmetry breaking and solvatochromism. J. Am. Chem. Soc. 128, 15742-15755 (2006).

47. Adarsh, N., Shanmugasundaram, M., Avirah, R. R. \& Ramaiah, D. Aza-BODIPY derivatives: Enhanced quantum yields of triplet excited states and the generation of singlet oxygen and their role as facile sustainable photooxygenation catalysts. Chem. Eur. J. 18(40), 12655-12662 (2012).

48. Silva, A. M. G., Tome, A. C., Neves, M. G. P. M. S. \& Cavaleiro, J. A. S. Porphyrins in 1,3-dipolar cycloaddition reactions: Synthesis of a novel pyrazoline-fused chlorin and a pyrazole-fused porphyrin. Synlett 7, 1155-1157 (2002).

49. Sasidharan, A. et al. Hemocompatibility and macrophage response of pristine and functionalized graphene. Small 8(8), 1251-1263 (2012).

50. Liu, H.-Y., Lei, Du., Zhao, Y.-T. \& Tian, W.-Q. In vitro hemocompatibility and cytotoxicity evaluation of halloysite nanotubes for biomedical application. J. Nanomater. https://doi.org/10.1155/2020/7435260 (2015).

\section{Acknowledgements}

This work was supported by the National Natural Science Foundation of China (No. 21977016), Foundation of Shanghai Science and Technology Committee (No. 18430713000, 18411968000, 18410721700, 18430731600, 19410711000, 19411970600, 20430730900, 20490740400).

\section{Author contributions}

F.S. has performed all experiments, wrote original draft. Y.-J.Y. reviewed and modified manuscript. D.M. provide funding Z.-L.C. supervised and reviewed the whole work.

\section{Competing interests}

The authors declare no competing interests.

\section{Additional information}

Supplementary Information The online version contains supplementary material available at https://doi.org/ 10.1038/s41598-021-89081-y.

Correspondence and requests for materials should be addressed to Y.-J.Y., D.M. or Z.-L.C.

Reprints and permissions information is available at www.nature.com/reprints.

Publisher's note Springer Nature remains neutral with regard to jurisdictional claims in published maps and institutional affiliations.

Open Access This article is licensed under a Creative Commons Attribution 4.0 International License, which permits use, sharing, adaptation, distribution and reproduction in any medium or format, as long as you give appropriate credit to the original author(s) and the source, provide a link to the Creative Commons licence, and indicate if changes were made. The images or other third party material in this article are included in the article's Creative Commons licence, unless indicated otherwise in a credit line to the material. If material is not included in the article's Creative Commons licence and your intended use is not permitted by statutory regulation or exceeds the permitted use, you will need to obtain permission directly from the copyright holder. To view a copy of this licence, visit http://creativecommons.org/licenses/by/4.0/.

(C) The Author(s) 2021 